\title{
Predictors of Neurological Presentations of COVID-19 Infected Patients in South Egypt, Aswan Governorate: A Single Center Study
}

\author{
Abeer A Tony (D' \\ Shazly BA Ahmed ${ }^{2}$ \\ Effat AE Tony ${ }^{3}$ \\ Salah Maklad ${ }^{4}$ \\ Islam Elnakeeb ${ }^{5}$ \\ Mohamed M Amin \\ 'Department of Neuropsychiatry, \\ Neurology Section, Faculty of Medicine, \\ Aswan University, Aswan, Egypt; \\ ${ }^{2}$ Department of Chest Diseases and TB, \\ Faculty of Medicine, Aswan University, \\ Aswan, Egypt; ${ }^{3}$ Department of Internal \\ Medicine, Faculty of Medicine, Assiut \\ University, Assiut, Egypt; ${ }^{4}$ Department of \\ Radiology, Faculty of Medicine, Aswan \\ University, Aswan, Egypt; ${ }^{5}$ Department of \\ Clinical Pathology, Faculty of Medicine, \\ Aswan University, Aswan, Egypt; \\ ${ }^{6}$ Department of Microbiology and \\ Immunology, Faculty of Medicine, Aswan \\ University, Aswan, Egypt
}

Background: COVID-19 is a complex multisystem disease comprising multiple organ dysfunctions including neurologic manifestations. Some COVID-19 patients may present neurologic symptoms as the initial presentations of the disease.

Objective: We aim at investigating the frequency and the predictors of neurological manifestations in patients with confirmed COVID-19.

Methods: A retrospective cross-sectional single-center study analyzed COVID-19 positive patients with neurological manifestations from March to June 2020, in Aswan Governorate, Egypt. Demographic data, clinical, radiological and laboratory findings, comorbidities, and treatments were collected and analyzed.

Results: Out of the 905 confirmed COVID-19 patients; 422 patients (46.6\%) had neurological manifestations and fulfilled the study inclusion criteria, 223 patients $(52.8 \%)$ had central neurological disorders (CNS), 107 (25.4\%) had peripheral neurological disorders (PNS), and $92(21.8 \%)$ patients had non-specific neurological disorders. Age $>50$ years, diabetes mellitus, CORAD> III and smoking were predictors for neurological system affection.

Conclusion: COVID-19 infection has been associated with numerous neurological deficits, especially in elderly patients. Central nervous system disorders were the most prevalent deficit with predominance of cerebrovascular events.

Keywords: COVID-19, central neurological disorders, CND, peripheral neurological disorders, PND

\section{Introduction}

It is already known that human Coronaviruses have neuroinvasive capabilities to spread from the respiratory tract to the Central Nervous System (CNS) through transneuronal and hematogenous routes. ${ }^{1,2}$ The latest studies demonstrated that, in severe cases, neurological manifestations may occur as a result of cerebral hypoxia due to respiratory failure. ${ }^{3}$ Some COVID-19 patients may present neurologic symptoms as initial presentations of the disease. ${ }^{4}$ Moreover, Taquet et al reported that COVID-19 was followed by significant rates of neurological and psychiatric diagnoses over the subsequent 6 months, which were greatest in patients who had severe COVID-19. ${ }^{5}$ Various mechanisms are proposed for explaining the possible CNS involvement of the SARS-COV-2 including direct invasion of the virus via disruption of blood brain barrier (BBB) through release of cytokines and retro- or anterograde neuronal transport via dynein and kinesins proteins. ${ }^{6}$ Moreover, platelet degranulation and activation
Correspondence: Abeer A Tony

Tel +201005389084

Fax $+2097-3480449$

Email abeer.tony@aswu.edu.eg 
occur due to loss of endothelium integrity as a result of entry of SARS-CoV-2 into endothelial cells. Serotonin, which is abundant in platelet granules and released upon degranulation, directly impacts blood vessel integrity and promotes leukocyte recruitment and cytokine release. ${ }^{7}$ SARS-COV-2 binding with angiotensin converting enzyme 2 (ACE2) receptors of capillary endothelium may disrupt the BBB and enter the CNS. Moreover, this binding leads to elevated blood pressure and increases the risk of cerebral hemorrhage. ${ }^{8,9}$ Additionally, SARS-COV-2 leads to both pneumonia and impairment of brain stem cardio-respiratory regulation center, which both lead to hypoxia and respiratory failure. ${ }^{10}$ This hypoxia may exacerbate the neural damage and support the higher prevalence of neurological symptoms in severely ill patients. Considering the lack of evidences and the importance of possible CNS roles in the COVID-19 pathophysiological, further studies are encouraged. Therefore, we believe that exploring the neurologic manifestations associated with COVID-19 is urgently required for better understanding of the SARS-CoV-2 brain infections, inhibiting the additional spread, treating patients affected by this pandemic and reducing the mortality rate in individuals infected with COVID-19. Therefore, we would like to highlight the neurological manifestations of the COVID 19 in Aswan Governorate, Egypt, and describe these neurological manifestations and their related demographic data.

\section{Aim of Study}

In this study, our aim is to describe the frequency of neurological symptoms in COVID-19 positive patients in Aswan Governorate, Egypt, clarify all the demographics associated with their presentations, and assess the predictors of these neurological manifestations and their different types.

\section{Methodology}

\section{Study Design}

This is a single-center retrospective cross-sectional study performed at Aswan University Hospitals (Chest Department, Emergency Unit and Neuropsychiatry Department) and AL-Sadaka Hospital (designated hospital assigned by the government to treat patients with COVID19), Aswan.

\section{Study Participants}

Study population included patients with age 18 years or older with a positive diagnosis of COVID-19 from March 2020, to June 2020. COVID-19 positive patients with neurological manifestations were enrolled. We picked out any neuropsychiatric diagnosis on, or within, one month after the diagnosis of COVID-19. Patients were distributed into two groups based on the severity of the respiratory symptoms (severe and non-severe) according to WHO and CDC Guidance for COVID-19. Severe respiratory symptoms were defined as respiratory insufficiency requiring mechanical ventilation. Patients were excluded if they had a history of previous neurological or psychiatric disorders to avoid the perplexing factor of accidental exacerbation.

\section{Data Collection and Study Strategy}

We reviewed electronic medical records, nursing records, laboratory findings, and radiologic examinations for all patients with laboratory-confirmed SARS-CoV-2 infection. All patients' medical information, including demographic characteristics, medical history including comorbidities (hypertension, diabetes, cardiac or cerebrovascular disease, malignancy, and chronic kidney disease), clinical, radiological (CT scan (chest and head if available) and/or MRI), cerebrospinal fluid (CSF) analysis, and electrophysiological studies, if were done, and laboratory findings (blood cell count, liver and renal function tests, C-reactive protein, D-dimer and ferritin levels), were documented. We defined the degree of severity of COVID-19 (severe versus non-severe) at the time of admission using WHO and CDC Guidance for COVID-19. ${ }^{11}$ Based on the chest CT findings, the level of suspicion of COVID-19 infection was graded from very low, or CO-RADS 1, up to very high, or CO-RADS 5, according to the COVID-19 Reporting and Data System (CO-RADS). ${ }^{12}$ All neurologic manifestations were reviewed and confirmed. They were categorized into central nervous system (CNS), peripheral nervous system (PNS) and non-specific neurological manifestations.

\section{PCR for Detection of SARS COV-2 Virus Blood Samples}

- $2 \mathrm{~mL}$ of venous blood was collected aseptically in EDTA tubes for CBC.

- $2 \mathrm{~mL}$ blood was collected aseptically in sodium citrate tubes for D-dimer.

- $3 \mathrm{~mL}$ venous blood was collected in plain tube for serum collection; the serum of the patients will be used for detection of CRP and ferritin. 


\section{Preparation of PCR}

According to the manufacturer's instructions QIAGEN (QIAamp-DSP virus spin kit, cat.no. 61,704 for purification of viral nucleic acid from nasopharyngeal and oropharyngeal swabs, three key steps were done: 1) sample collection, 2) RNA extraction, and 3) viral RNA detection.

Sample collection: was done using a swab to collect respiratory material found in the nasopharynx and oropharynx. A swab contains a soft tip on a long, flexible stick that was inserted into the nose and collected a sample immediately. After collection, swab was placed in $\sim 3 \mathrm{~mL}$ of viral transport medium and transported to the clinical lab for diagnostic testing, within 4 hours of collection. When the sample was received, an extraction was performed, which isolated genetic material from the sample including genetic material from any virus that may be present.

RNA extraction: according to the manufacturer's instructions QIAGEN

1. $10 \mu \mathrm{L}$ Proteinase K, $300 \mu \mathrm{L}$ lyses buffer DVN and 4 ul of poly A (lysis mix) were added to a clean $1.5 \mathrm{~mL}$ RNase-Free Microcentrifuge Tube. Then we added $200 \mu \mathrm{L}$ of sample (nasopharyngeal/oropharyngeal swab) to the centrifuge tube, mixed by vortexing for $15 \mathrm{sec}$ and incubated at room temperature for $5 \mathrm{~min}$.

2. The lysate was carefully transferred onto the Fine Bind DNA Spin Columns in a $2 \mathrm{~mL}$ Collection Tube. The cap was closed and centrifuged at 10,000 rpm for $1 \mathrm{~min}$, the filtrated was discarded and the spin column was placed in the same collection tube.

3. The Fine Bind DNA Spin Columns was opened carefully, and $700 \mu \mathrm{L}$ Buffer DW1P was added. The cap was then closed and centrifuged at $10,000 \mathrm{rpm}$ for $1 \mathrm{~min}$. The filtrated was discarded and the spin column was placed in the same collection tube.

4. The Fine Bind DNA Spin Columns were carefully opened and $700 \mu \mathrm{L}$ Buffer MWP was added. The cap was closed and centrifuged at 10,000rpm for $1 \mathrm{~min}$. The filtrated was discarded and the spin column was placed in the same collection tube.

5. Centrifuge at full speed $12,000 \mathrm{rpm}$ for 2 min to dry the membrane completely.

6. The Fine Bind DNA Spin Columns were placed in a clean $1.5 \mathrm{~mL}$ RNase-Free Microcentrifuge Tubes, the lid of the spin column was opened carefully. $100 \mu \mathrm{L}$ of RNase-Free ddH2O was added to the center of the membrane. The lid was closed, incubated, and centrifuged at 10,000 rpm for $1 \mathrm{~min}$.

7. In this protocol, carrier RNA was diluted to a volume of $25 \mu \mathrm{L}$ per sample in Buffer AVE. We prepared carrier RNA for the required number of samples and plus three additional samples (eg, for 7 samples, prepare carrier RNA for 10 samples). Prepare $25 \mu \mathrm{L}$ carrier RNA as described below:

A. No internal control: Carrier RNA: $5.6 \mu \mathrm{L}$ and Buffer AVE: $19.4 \mu \mathrm{L}$

B. With internal control: Carrier RNA: $5.6 \mu \mathrm{L}$ and Internal control: $\mathrm{Up}$ to $19.4 \mu \mathrm{L}$ (if $<19.4 \mu \mathrm{L}$ internal control is used, adjust final volume to25 $\mu$ l with Buffer AVE)

Viral RNA Detection: by real-time PCR, which causes a reaction that makes millions of copies of a small portion of the SARS-CoV-2 virus's genetic material, was done.

1. 15 ul of rehydration buffer was put in each PCR tube.

2. 5 ul of elution buffer was put in sample tubes.

3. The tube caps were closed.

4. Positive control was prepared by mixing $100 \mathrm{ul}$ of nuclease free water in positive control tube.

5. $5 \mathrm{ul}$ of prepared positive control reagent was used instead of sample with addition of rehydration buffer as before.

6. Negative control preparation was made by mixing 15 ul of rehydration buffer with 5 ul of negative control.

\section{Standard Protocol Approvals, Registrations, and Patient Consents}

This study was approved by local institutional ethical committees. Approval for the study was obtained from the IRB Committee, Faculty of Medicine, Aswan University (EC Ref No.: aswu/485/10/20; Date: 11/10/ 2020). Permission from the dataset owner to use the information in databases/repositories for the purposes of the research was provided. The authors report no disclosures relevant to the manuscript. There are no reports on clinical trial, and no clinical trial registry.

\section{Ethical Consideration}

The current study was approved by the Institutional Ethics Committee, Faculty of Medicine, Aswan University (EC 
Ref No.: aswu/485/10/20; Date: 11/10/2020). Written informed consent was waived as this retrospective study was carried out to investigate an emerging infectious disease. Permission from the dataset owner to use the information in databases/repositories for the purposes of the research was provided. The study was conducted in accordance with the principles of Declaration of Helsinki.

\section{Data Availability}

Data related to the current article are available from Abeer A. Tony, Assistant Professor of Neurology, Aswan University. This is in accordance with the data-protection legislation in Europe (General Data Protection Regulation). Persons interested in obtaining access to the data should contact Abeer A. Tony (abeer.tony@aswu.edu. eg). The study complies with relevant patient data confidentiality regulations.

\section{Statistical Analysis}

A statistical analysis was conducted using the Statistical Package for the Social Sciences (IBM-SPSS) version 19. Data were expressed as medians with the interquartile range (IQR) or means \pm standard deviation (SD), according to the distribution. Chi-square test was used to compare frequency between groups. Student's $t$-test (normal distributions), or Mann-Whitney test (non-normal distributions) was used to analyze continuous variables. Multivariate regression analysis was used to determine the independent risk factors for prediction of central and peripheral nervous system affections in the studied patients. P value was significant if $<0.05$.

\section{Results}

Basic Characteristics of our studied confirmed COVID-19 patients are shown in Figure 1A-C. Figure 2 shows the types of neurological manifestations observed in our patients. The distribution of central and peripheral neurological disorders was shown in Figures 3 and 4. Regarding the comparison between the clinical characteristics of our confirmed COVID-19 patients with CNS and those with PNS manifestations, it is presented in Table 1. Male gender was predominated in patients with CNS (70\%), while the majority of patients with PNS were females (52.3\%) with a significant statistical difference $(\mathrm{p}<0.01)$. The majority of COVID-19 positive patients with CNS (100; 44.8\%) were at the age of 60 years and above, while our patients with PNS $(30 ; 28 \%)$ were at the middle age with a significant statistical difference $(p<0.01)$. Regarding the risk factors, smoking and HTN were significantly the commonest risk factors in patients with CNS, while in patients with PNS, the commonest risk factors were smoking and DM with a statistical difference $\mathrm{p}<0.01$. Significantly, our patients with CNS had the most severe pulmonary affection (CO-RAD IV and CO-RAD V in $36.3 \%$ and $26.5 \%$ respectively). Conversely, minor pulmonary findings (CO-RAD II and CO-RAD I in 54.2\% and 29\% respectively) were predominant in patients with PNS ( $p<$ 0.01 ). Interestingly, a greater number of our confirmed COVID-19 patients with CNS manifestations had higher levels of positive CRP, D-Dimer and Ferritin (67.3\%, $20.2 \%$ and $39.9 \%$ respectively) when compared to those with PNS diseases, but with no statistical difference, as shown in Table 1. Based on the current study, predictors of neurological affection in patients with COVID-19 were aged $>50$ years, DM, smoking and CO-RAD $>$ III $(p<0.001$ for each) with special reference to the central nervous disorders, as shown in Tables 2 and 3. Meanwhile, dyslipidemia and positive CRP ( $\mathrm{p}=0.03$ and 0.02 respectively) were the predictors of peripheral nervous system affection in patients with COVID-19, as shown in Table 4.

\section{Discussion}

COVID-19 is a complex multisystem disease comprising multiple organ dysfunctions, which include neurologic manifestations. The neurologic symptoms may be the initial or the only presentations of the COVID-19., ${ }^{4,13}$ In the present study, we reported neurologic symptoms in $46.6 \%$ of COVID-19 positive patients. They had the following characteristics: male sex predominance, older age (60 years and above), more prevalence of smoking and HTN as risk factors with severe pulmonary affection. Our result was matched with other studies in China and Europe. ${ }^{14,15}$ Nalleballe et al, found that $22.5 \%$ of the patients have neuropsychiatric manifestations related to COVID-19. ${ }^{16}$ Our result was contradictory to the results of Anand et al, who reported that 8\% of patients with positive COVID-19 had inpatient neurologic admissions. ${ }^{17}$ High heterogeneity in our study could be caused by differences in severity of the disease, associated co-morbidities, ethnicity of patients, and variation in the number of patients or due to differences in the methodology among the studies. Significantly, the higher prevalence of neurological signs and symptoms observed among our patients with serious pulmonary affection, was parallel to the observation of previous studies. ${ }^{4,10,14,15}$ Hypoxia resulted from impairment of brain stem cardio-respiratory regulation center due to SARS-COV-2 infection, exacerbates the neural 


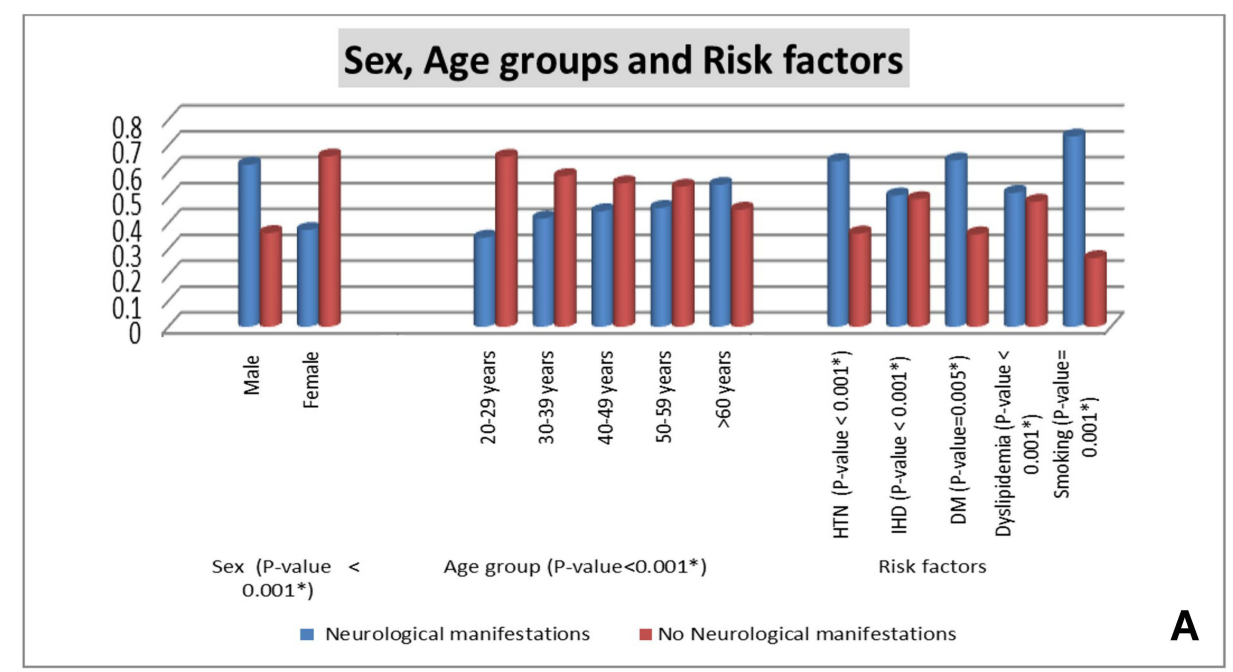

HTN; hypertension, IHD; ischemic heart disease, DM; diabetes mellitus. P-value is considered significant if $<0.05 .{ }^{*}$ Chi-square test was used

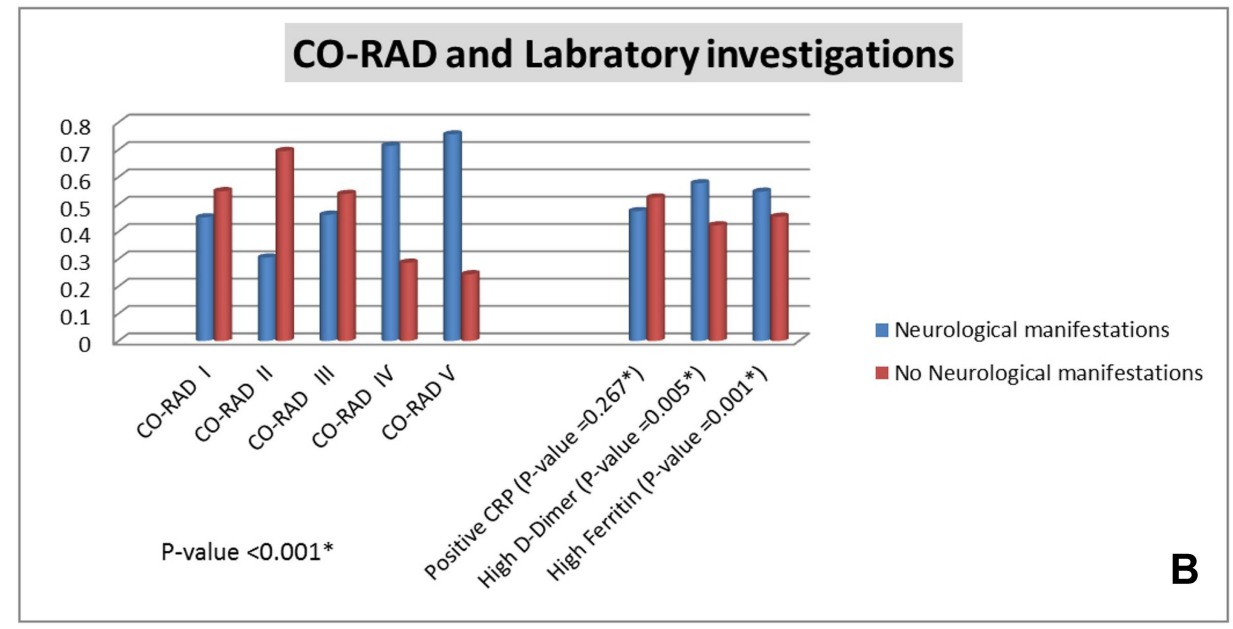

P-value is considered significant if $<0.05 .{ }^{*}$ Chi-square test was used

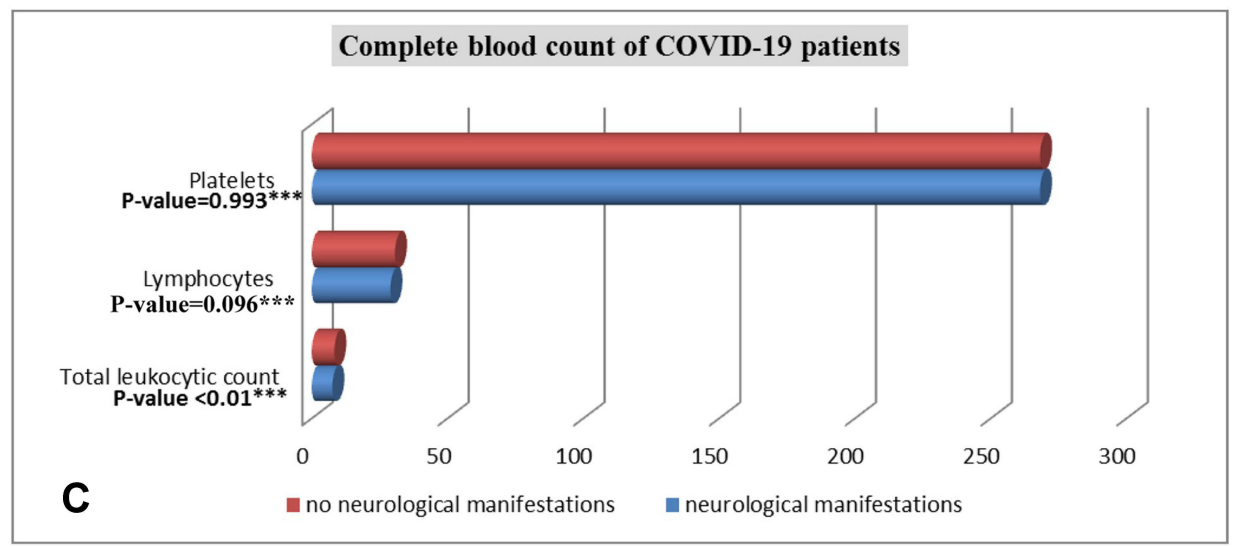

P-value is considered significant if $<0.05 .^{* * *}$ Mann-Whitney test was used

Figure I Basic characteristics of studied confirmed COVID-19 patient (A-C). 


\section{Distribution of neurological disorders (46.6\%)}
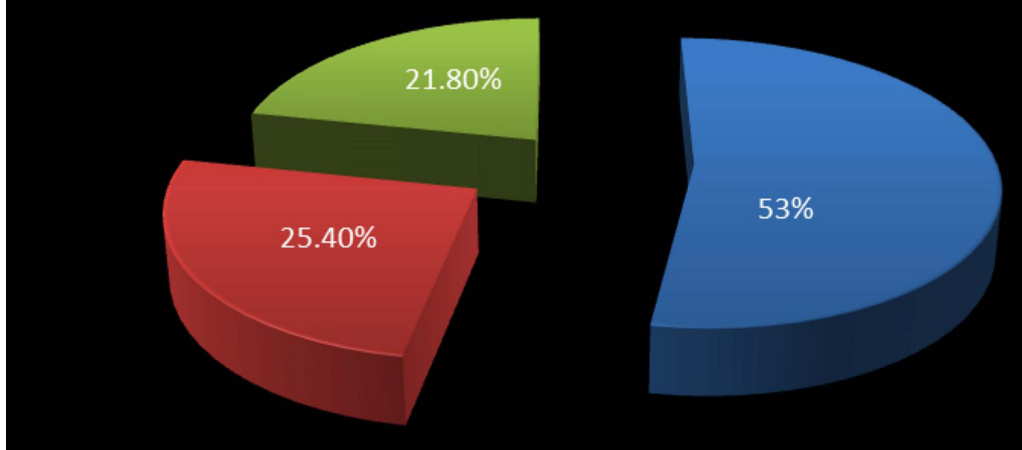

× COVID-19 positive Patients with CNS manifestations

× COVID-19 positive Patients with PNS manifestations

m COVID-19 positive Patients with nonspecific neurological manifestations

Figure 2 Distribution of neurological manifestations among our COVID-19 confirmed patients. Non-specific neurological manifestations (Myalgia, headache, and either anosmia, ageusia or anosmia and ageusia).

Abbreviations: CNS; central nervous system, PNS; peripheral nervous system.

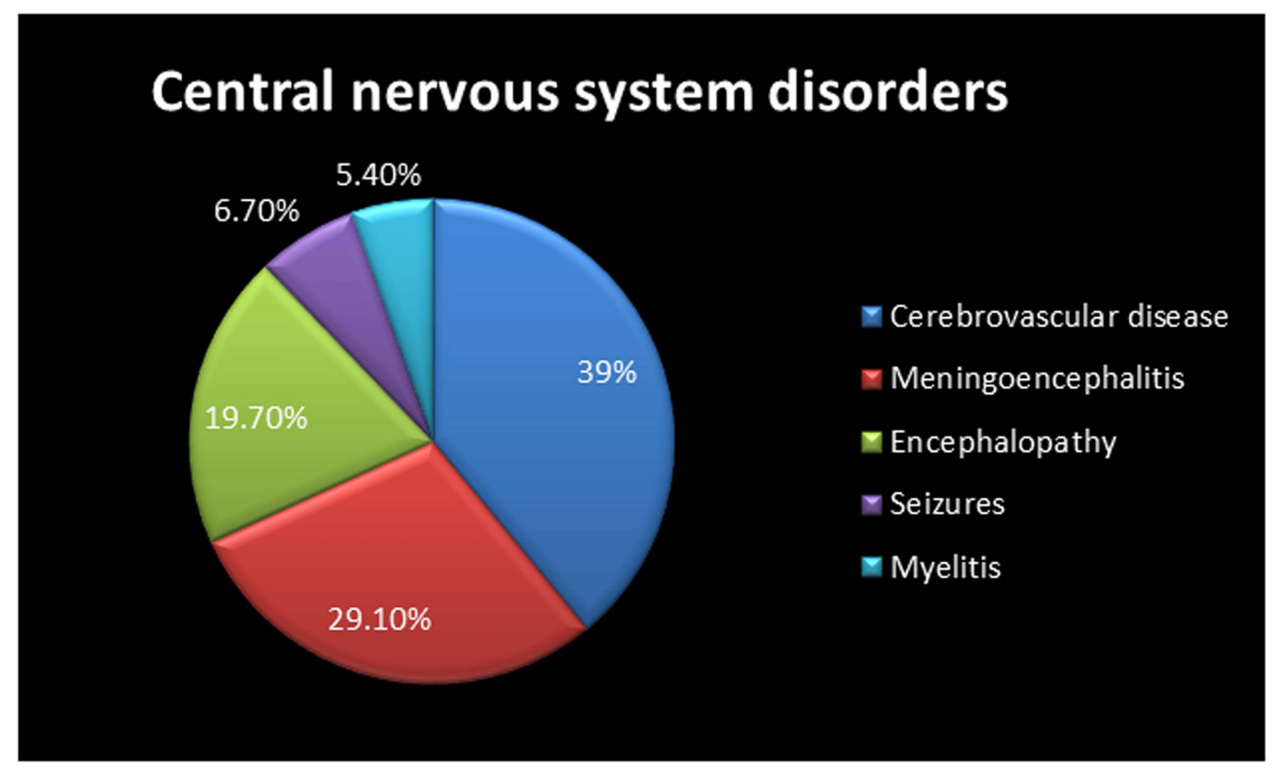

Figure 3 Distribution of central neurological manifestations among our COVID-19 confirmed patients.

damage. Furthermore, the blood-brain-barrier (BBB) normally hinders virus entry but is impaired in some cases by inflammatory conditions and infection of brain endothelial cells. ${ }^{18}$ Potential mechanisms of neuropsychiatric manifestation include direct CNS infiltration, cytokine network dysregulation, peripheral immune cell transmigration, and post-infectious autoimmune response. ${ }^{16}$ A growing body of evidence shows that neuroinvasion and neurotropism are common features of human Coronaviruses. The neuroinvasive human viruses and the respiratory viruses may damage the CNS as a result of misdirected host immune responses that could be associated with autoimmunity in susceptible individuals (virus-induced neuroimmunopathology) and/or viral replication, which directly causes damage to CNS 


\section{Peripheral nervous system disorders}

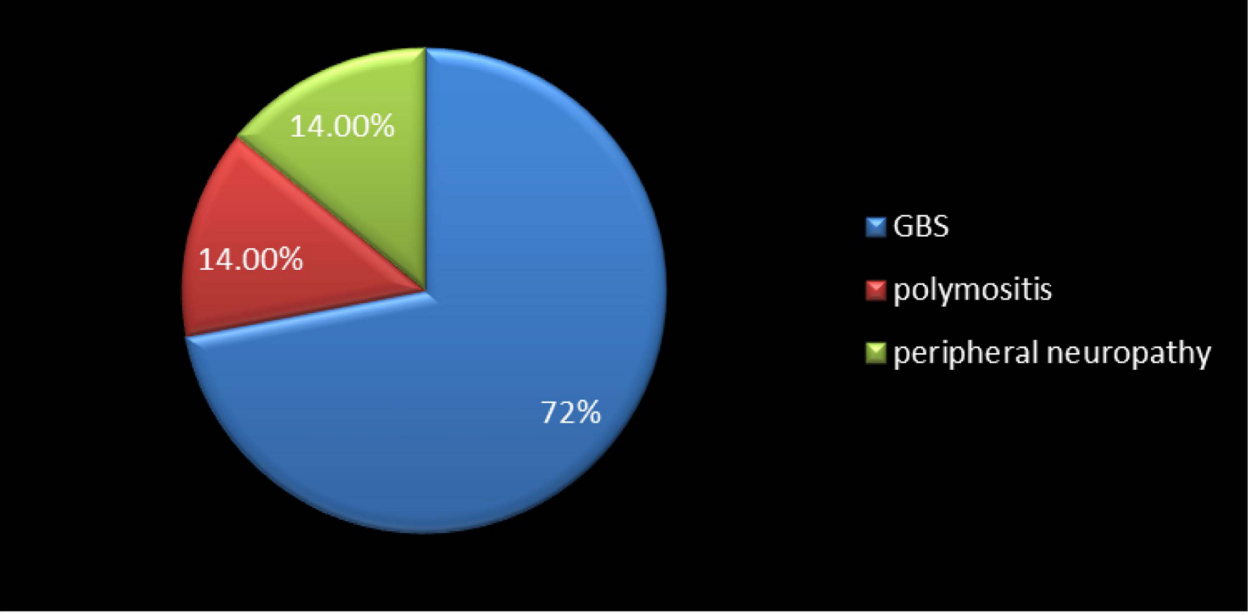

Figure 4 Distribution of peripheral neurological manifestations among our COVID-19 confirmed patients.

cells (virus-induced neuropathology). ${ }^{19}$ In our study, the acute CND were represented in more than half of our population, which was synchronized with the reports of Mao and co-workers. ${ }^{14}$ Notably, cerebrovascular diseases were the commonest central neurological disorders among our studied patients. The majority of them had ischemic stroke. Our results were similar to the reports of others. ${ }^{6,20,21}$ Down regulation of natural anticoagulant mechanisms by inflammatory mediators and disturbance of coagulation system is the proposed pathophysiological mechanism for the occurrence of ischemic stroke. ${ }^{22}$ Moreover, Mao et al, found that patients with severe infection had higher D-dimer levels than that of patients with none severe infection. ${ }^{14}$ This may be the reason why patients with severe infection are more likely to develop cerebrovascular disease. Additionally, it is important to note that accumulating evidence shows that the blood of COVID-19 patients is hypercoagulable. ${ }^{23-25}$ Intracerebral hemorrhage was observed in $34.5 \%$ of our patients with CVD. Although reports of ICH associated with COVID-19 are scarce that had experienced small percentage of patients with cerebral hemorrhage in their studies. ${ }^{8,26}$ This difference could be explained by the large size sample of our patients, and the higher prevalence of hypertension and smoking as risk factors among them. Following SARS-CoV-2 infection, the expression and function of ACE2 proteins are reduced, and since the expression of ACE2 in patients with hypertension is already low, SARS-CoV-2 infection is more likely to induce cerebral hemorrhage in such patients. ${ }^{27}$ Another potential etiology is endothelial dysfunction. ACE II receptors are expressed in cerebrovascular endothelial cells and regulate the sympathoadrenal system, vascular autoregulation, and cerebral blood flow. ${ }^{28}$ Virus binding to the CNS ACE II receptors can lead to disruption of its autoregulatory function, causing blood pressure elevations leading to vessel wall rupture. ${ }^{8}$ Older individuals, especially those with preexisting chronic medical conditions, are at an elevated risk of impaired consciousness or delirium in the setting of acute infections. These patients, who are prone to experience COVID-19 severely, may present with encephalopathy and confusion. $^{29}$ In the present study, $19.7 \%$ of the cases had features consistent with encephalopathy. Encephalopathy might be due to direct invasion of the CNS by SARS-CoV -2 , inflammation secondary to a cytokine storm, or as a result of septic encephalopathy. Our result was in agreement with Poyiadji et al, who stated that encephalopathy had been identified as one of the symptoms of COVID-19. ${ }^{30}$ In contrast, Nalleballe et al, detected encephalopathy in $2.3 \%$ of patients with positive COVID-19 who explained this by the presence of metabolic abnormalities from multi organ involvement with a possibly contributory role from nervous system involvement by the virus. ${ }^{16}$ Furthermore, $29.1 \%$ of our cases had features consistent with meningo-encephalitis. Our result was parallel to numerous studies. ${ }^{31-35}$ Virus-induced immune response leading to inflammatory damage of the CNS and direct invasion (viral neurotropism) are the two main pathophysiological mechanisms of SARS-CoV-2-associated encephalitis/meningitis. ${ }^{35} \mathrm{DE}$ novo seizures were presented in $6.7 \%$ of our patients with CNS manifestations. This 
Table I Comparative Criteria Between of COVID-I9 Positive Patients with CND and Those with PND

\begin{tabular}{|c|c|c|c|}
\hline Parameter & $\begin{array}{l}\text { Patients with CND } \\
(\mathrm{No}=223 ; 52.8 \%)\end{array}$ & $\begin{array}{l}\text { Patients with PND } \\
\text { (No = 107; 25.4\%) }\end{array}$ & P-value \\
\hline $\operatorname{Sex}(M / F)(N o ; \%)$ & $156 / 67(70.0 \% / 30.0 \%)$ & $5 \mathrm{I} / 56(47.7 \% / 52.3 \%)$ & $<0.001 *$ \\
\hline \multicolumn{4}{|l|}{ Age group (No; \%) } \\
\hline 20-29 years & II (4.9\%) & $16(15.0 \%)$ & \\
\hline $30-39$ years & $24(10.8 \%)$ & $30(28.0 \%)$ & \\
\hline $40-49$ years & $42(18.8 \%)$ & $25(23.4 \%)$ & $<0.00 I^{*}$ \\
\hline $50-59$ years & $46(20.6 \%)$ & $21(19.6 \%)$ & \\
\hline$>60$ years & $100(44.8 \%)$ & 15 (I4.0\%) & \\
\hline \multicolumn{4}{|l|}{ Risk factors (No; \%) } \\
\hline Hypertension & $120(53.8 \%)$ & 24 (22.4\%) & $<0.00 I^{*}$ \\
\hline Ischemic heart disease & 117 (52.5\%) & $23(21.5 \%)$ & $<0.001 *$ \\
\hline Diabetes mellitus & $108(48.4 \%)$ & 35 (32.7\%) & $=0.005^{*}$ \\
\hline Dyslipidemia & 97 (43.5\%) & $16(15.0 \%)$ & $<0.001 *$ \\
\hline Smoking & $129(57.8 \%)$ & $4 \mathrm{I}(38.3 \%)$ & $=0.001 *$ \\
\hline \multicolumn{4}{|l|}{ CO-RAD (No; \%) } \\
\hline CO-RAD I & 19 (8.5\%) & $31(29.0 \%)$ & \\
\hline CO-RAD II & 16 (7.2\%) & $58(54.2 \%)$ & \\
\hline CO-RAD III & $48(21.5 \%)$ & $10(9.3 \%)$ & $<0.001 *$ \\
\hline CO-RAD IV & 81 (36.3\%) & 7 (6.5\%) & \\
\hline CO-RAD V & 59 (26.5\%) & I (0.9\%) & \\
\hline \multicolumn{4}{|l|}{ Laboratory data } \\
\hline \multicolumn{4}{|l|}{ A. $\mathrm{CBC}($ mean+SD) } \\
\hline Hemoglobin & $12.152 \pm 1.612$ & $11.938 \pm 1.616$ & $=0.262^{* *}$ \\
\hline Total leukocyte count & $7.418 \pm 3.414$ & $7.933 \pm 3.548$ & $=0.206 * * *$ \\
\hline Lymphocytes & $28.423 \pm 14.764$ & $28.178 \pm 13.274$ & $=0.884 * * *$ \\
\hline Platelets & $265.87 \pm 85.206$ & $269.19 \pm 88.700$ & $=0.744 * * *$ \\
\hline B. Positive CRP (No; \%) & $150(67.3 \%)$ & 79 (73.8\%) & $=0.139 *$ \\
\hline C. High D-Dimer (No; \%) & $45(20.2 \%)$ & $30(28.0 \%)$ & $=0.074 *$ \\
\hline D. High Ferritin (No; \%) & 89 (39.9\%) & 38 (35.5\%) & $=0.259 *$ \\
\hline
\end{tabular}

Notes: The bold formatting in the table means significant $\mathrm{P}$-value; P-value is considered significant if $<0.05$; *Chi-square test was used; **Student's $t$-test was used; ***MannWhitney test was used.

Abbreviations: CVD, central nervous disorder; PND, peripheral nervous disorder; M, Male; F, Female; CO-RAD, COVID-I9 Reporting and Data System; CBC, Complete blood count; CRP, C-reactive protein; No, Number.

was in concordance with others. ${ }^{29-38}$ Decreased seizure thresholds that triggering neuronal hyperexcitability from cytokine surge might be the underlying mechanism for COVID-19 related seizures. Other proposed hypotheses included a consequence of hypoxia, metabolic derangements, or even neuroinvasion that may happen to these patients. ${ }^{2}$ In the current study, transverse myelitis was observed in 5.4\% of patients, in agreement with other literature. ${ }^{39-42}$ It was probably due to heightened inflammation following a cytokine storm brought on by COVID-19. ${ }^{30}$ Nevertheless, it is still debatable whether the myelitis occurs directly from the viral infection, or as an autoimmune sequelae. ${ }^{41}$ In the present study, the acute PND were represented in $25.4 \%$ of our population which was synchronized with the reports of
Mao et al. ${ }^{14}$ They had the following characteristics: female sex predominance, middle age, more predominance of smoking and DM as risk factors with minor pulmonary affection. Remarkably, GuillainBarré syndrome (GBS) was the commonest peripheral neurological disorders (71.9\%) among our studied patients. Pathogen-associated antibodies that attack peripheral nerves due to molecular mimicry have been previously put forward as a disease mechanism in GBS. However, Finsterer et al, reported that none of the patients with post-COVID-19 GBS tested positive for SARS-CoV-2 in the CSF, pointed to an immune mechanism such as inflammation secondary to a cytokine storm as a possible cause. ${ }^{42}$ Moreover, Zhao et al, associated Covid-19 viral disease with Guillain-Barré syndrome where neurological symptoms 
Table 2 Predictors of Neurological Affection in Patients with COVID-19 Infection

\begin{tabular}{|l|c|c|c|}
\hline & Odd's Ratio & $\mathbf{9 5 \%} \mathbf{C l}$ & P value \\
\hline Age (>50 years) & 2.01 & 1.28 to 3.15 & $<\mathbf{0 . 0 0 I}$ \\
Sex & 1.27 & 0.72 to 2.21 & 0.39 \\
DM & 4.03 & 2.44 to 6.65 & $<\mathbf{0 . 0 0 I}$ \\
Dyslipidemia & 0.62 & 0.39 to 0.99 & 0.05 \\
Hypertension & 1.06 & 0.64 to 1.74 & $0.8 \mathrm{I}$ \\
Ischaemic heart disease & 0.71 & 0.44 to 1.13 & 0.15 \\
Smoking & 4.41 & 2.54 to 7.66 & $<\mathbf{0 . 0 0 I}$ \\
Leukocytosis & 0.66 & 0.39 to 1.09 & 0.11 \\
Positive CRP & 1.19 & 0.78 to 1.83 & 0.40 \\
Positive d-dimer & 1.29 & 0.78 to 2.13 & $0.3 \mathrm{I}$ \\
Positive ferritin & 1.15 & 0.77 to 1.72 & 0.48 \\
CORD> III & 15.27 & 10.05 to 23.22 & $<\mathbf{0 . 0 0 I}$ \\
\hline
\end{tabular}

Notes: P-value is significant if $<0.05$; The bold formatting in the table means significant $\mathrm{P}$-value.

Abbreviations: $\mathrm{Cl}$, confidence interval; $\mathrm{AF}$, atrial fibrillation; $\mathrm{DM}$, diabetes mellitus; CRP, C-reactive protein.

Table 3 Predictors of Central Neurological Affection Among Patients with COVID-19

\begin{tabular}{|l|c|c|c|}
\hline & Odd's Ratio & $\mathbf{9 5 \%} \mathbf{~ C I}$ & P value \\
\hline Age (>50 years) & 2.24 & 1.39 to 3.60 & $<\mathbf{0 . 0 0 I}$ \\
Sex & 1.20 & 0.67 to 2.14 & 0.53 \\
DM & 3.95 & 2.33 to 6.70 & $<0.00$ I \\
Dyslipidemia & 0.74 & 0.46 to 1.20 & 0.23 \\
Hypertension & 1.19 & 0.7 I to $2.0 \mathrm{I}$ & 0.49 \\
Ischaemic heart disease & 0.65 & 0.40 to 1.07 & 0.09 \\
Smoking & 4.23 & 2.37 to 7.53 & $<\mathbf{0 . 0 0 I}$ \\
Leukocytosis & 0.63 & 0.37 to 1.07 & 0.08 \\
Positive CRP & 1.50 & 0.95 to 2.36 & 0.07 \\
Positive d-dimer & 1.33 & 0.78 to 2.26 & 0.28 \\
Positive ferritin & 1.16 & 0.76 to 1.77 & 0.46 \\
CORD> III & 20.97 & 13.30 to 33.06 & $<\mathbf{0 . 0 0 I}$ \\
\hline
\end{tabular}

Notes: P-value is significant if $<0.05$; The bold formatting in the table mean significant $\mathrm{P}$-value.

Abbreviations: $\mathrm{Cl}$, confidence interval; $\mathrm{AF}$, atrial fibrillation; $\mathrm{DM}$, diabetes mellitus; CRP, C-reactive protein.

occurred without any preceding respiratory symptoms suggesting a para-infectious rather than the post-infectious neuronal injury. ${ }^{39}$ Favas et al, reported that possible pathogenesis of GBS in COVID-19 included immune dysregulation secondary to systemic hyper inflammation and cytokines produced. ${ }^{43}$ Interestingly, peripheral neuropathy and polymyositis were equally distributed in our studied patients. Nalleballe et al, informed that polyneuropathy was secondary to direct viral invasion precipitating demyelination versus immune mediated damage from inflammatory cytokine surge. ${ }^{16}$ Furthermore, Zhang et al reported a patient with
Table 4 Predictors of Peripheral Neurological Affection Among Patients with COVID-19

\begin{tabular}{|l|c|c|c|}
\hline & Odd's Ratio & $\mathbf{9 5 \%} \mathbf{C l}$ & $\mathbf{P}$ value \\
\hline Age (>50 years) & 0.70 & 0.21 to 2.33 & 0.56 \\
Sex & 2.10 & 0.344 to $I 2.85$ & 0.42 \\
DM & 2.23 & 0.60 to 8.23 & 0.22 \\
Dyslipidemia & 1.09 & 1.01 to $I .87$ & $\mathbf{0 . 0 3}$ \\
Hypertension & 0.40 & 0.07 to 2.33 & 0.31 \\
Ischaemic heart disease & 1.49 & 0.40 to 5.49 & 0.54 \\
Smoking & 2.96 & 0.53 to 16.36 & 0.21 \\
Leukocytosis & 0.87 & 0.16 to 4.68 & 0.87 \\
Positive CRP & 1.26 & 1.08 to 2.87 & $\mathbf{0 . 0 2}$ \\
Positive d-dimer & 0.82 & 0.17 to 3.99 & 0.81 \\
Positive ferritin & 1.06 & 0.30 to 3.70 & 0.91 \\
CORD> III & 0.23 & 0.04 to 1.12 & 0.07 \\
\hline
\end{tabular}

Notes: P-value is significant if $<0.05$; The bold formatting in the table means significant $\mathrm{p}$-value.

Abbreviations: $\mathrm{Cl}$, confidence interval; $\mathrm{AF}$, atrial fibrillation; DM, diabetes mellitus; CRP, C-reactive protein.

COVID-19 associated myositis. ${ }^{44}$ COVID-19 is associated with a viral myositis attributable to direct myocyte invasion or induction of autoimmunity. Virus-mediated muscle inflammation is attributed to ACE2 receptor-mediated direct entry and affliction of muscle fibers, leading to innate and adaptive immune activation. ${ }^{45}$ Essentially, the present analysis found that current smokers have an increased risk of presenting neurological symptoms which is more noticeable to central nervous system presentations. This was in concordance with Reddy et $\mathrm{al}^{26}$ who confirmed that ACE-2 expression is upregulated in human lung tissue samples taken from both current and past smokers, likely mediated by the $\alpha-7$ subtype of the nicotinic acetylcholine receptor. ${ }^{46-49}$ Moreover, Smith et al, reported a consistent correlation between smoking history and increased ACE-2 expression. ${ }^{50}$ It is, therefore, plausible that smokers are exposed to higher SARS-CoV-2 loads as a result of increased expression of ACE-2, which may provide a mechanistic explanation for the increased risk of severe disease and mortality associated with smoking in COVID-19 patients. Other risk factors as hypertension, diabetes mellitus, and dyslipidemia were significantly more prevalent in our positive COVID-19 cases with neurological deficits. In agreement with Anand et al, higher D-dimer, ferritin and CRP levels were observed in our patients with CNS manifestations compared to those with PNS diseases. ${ }^{17}$ In contradictory, there was no statistically difference in lymphocyte count in both groups of patients. Azhideh ${ }^{3}$ noted lower lymphocyte and platelet counts in patients with severe CNS involvement, 
while laboratory findings may not be helpful in patients with PNS involvement. In addition, he found that the lymphocyte counts were lower for patients with CNS symptoms than without CNS symptoms. This phenomenon may be indicative of the immunosuppression in patients with COVID-19 with CNS symptoms, especially in the severe subgroup. In the current study, increased ages, diabetes mellitus, smoking and CORAD > III were considered as predictors for neurological affection in patients with COVID-19 infection accusing the role of these factors in central nervous system diseases. Direct viral infection of the CNS, inflammatory (cytokine storm), prothrombotic, and endothelial (via ACE2) pathways, hypoxia, systemic and/or metabolic disturbances like DM, and aging appear to be the predominant mechanisms for CNS involvement. Nevertheless, the predictors for peripheral nervous system manifestations were dyslipidemia and increased CRP reflecting the impact of immune- mediated pathogenesis. Tang et al, ${ }^{51}$ concluded that lipid disturbances were associated with demyelinating polyneuropathy in GBS patients suggesting impaired myelin membrane integrity in GBS patients. We acknowledge several limitations to our study. First, due to the observational retrospective nature of this study, ancillary testing was not standardized across all patients, with variable investigations performed. Second, this study was cross-sectional in design, and the data were collected retrospectively. Furthermore, we did not have access to the whole database and information. Finally, the focus of this study was on acute neurologic findings in hospitalized patients, without analysis of posthospital complications or mortality.

\section{Conclusion}

The current study showed that Central Nervous System disorders were the most prevalent deficit with predominance of cerebrovascular events. With a rapidly rising toll of COVID-19 patients with neurological manifestations, there is an urgent need to understand and diagnose the neurological symptoms earlier to highlight the treatment protocols on basis of severity of the disease. Moreover, given the crucial role of neuropathology of SARS-CoV-2, future therapies should target neurotropism of the virus and combine anti-neuroinflammatory and neuroregenerative features. Further studies will be needed to characterize the long-term neurological and psychiatric sequelae of SARS-CoV-2 infection, which needs serious consideration and follow-up tracking studies.

\section{Abbreviations}

COVID-19, Coronavirus Disease 2019; CORAD, COVID19 Reporting and Data System; SARS-CoV-2, Severe Acute Respiratory Syndrome Coronavirus 2; ACE2, Angiotensin Converting Enzyme 2; BBB, blood brain barrier; WHO, The World Health Organization.

\section{Acknowledgments}

We thank all subjects for their participation and acceptance to be studied in our work.

\section{Funding}

There is no funding to report.

\section{Disclosure}

The authors report no conflicts of interest for this work.

\section{References}

1. Desforges M, Le Coupanec A, Dubeau P, et al. Human coronaviruses and other respiratory viruses: underestimated opportunistic pathogens of the central nervous system? Viruses. 2020;12(1):14. doi:10.3390/ v12010014

2. Asadi-Pooya AA, Simani L. Central nervous system manifestations of COVID-19: a systematic review. J Neurol Sci. 2020;413:116832. doi:10.1016/j.jns.2020.116832

3. Azhideh A. COVID-19 neurological manifestations. Int Clin Neurosci J. 2020;7(2):54. doi:10.34172/icnj.2020.01

4. Jiang F, Deng L, Zhang L, Cai Y, Cheung CW, Xia Z. Review of the clinical characteristics of coronavirus disease 2019 (COVID-19). J Gen Intern Med. 2020;35(5):1545-1549. doi:10.1007/s11606-020-05762-w

5. Taquet M, Geddes JR, Husain M, Luciano S, Harrison PJ. 6-month neurological and psychiatric outcomes in 236379 survivors of COVID-19: a retrospective cohort study using electronic health records. Lancet Psychiatry. 2021;8(5):416-427. doi:10.1016/S22150366(21)00084-5

6. Wu Y, Xu X, Chen Z, et al. Nervous system involvement after infection with COVID-19 and other coronaviruses. Brain Behav Immun. 2020. doi:10.1016/j.bbi.2020.03.031

7. Zaid Y, Puhm F, Allaeys I, et al. Platelets can contain SARS-CoV-2 RNA and are hyperactivated in COVID-19. Circ Res. 2020;127 (11):1404-1418. doi:10.1161/CIRCRESAHA.120.317703

8. Sharifi-Razavi A, Karimi N, Rouhani N. COVID-19 and intracerebral haemorrhage: causative or coincidental? New Microbes New Infect. 2020;35:100669. doi:10.1016/j.nmni.2020.100669

9. Liu Z, Xiao X, Wei X, et al. Composition and divergence of coronavirus spike proteins and host ACE2 receptors predict potential intermediate hosts of SARS-CoV-2. J Med Virol. 2020;92(6):595-601. doi: $10.1002 / \mathrm{jmv} .25726$

10. Steardo L, Zorec R, Verkhratsky A. Neuroinfection may potentially contribute to pathophysiology and clinical manifestations of COVID-19. Acta Physiol. 2020;12(1):14. doi:10.1111/apha.13473

11. National Institutes of Health. Coronavirus disease 2019 (COVID-19) treatment guidelines; 2021. Available from: www.covid19.treatment. guidelines.nih.gov. Accessed May 12, 2021.

12. Prokop M, van Everdingen W, van Rees Vellinga T, et al. CO-RADS: a categorical CT assessment scheme for patients suspected of having COVID-19-definition and evaluation. Radiology. 2020;296:2. doi:10.1148/radiol.2020201473 
13. Bai Y, Yao L, Wei T, et al. Presumed asymptomatic carrier transmission of COVID-19. JAMA. 2020;323:1406. doi:10.1001/ jama.2020.2565

14. Mao L, Jin H, Wang M, et al. Neurologic manifestations of hospitalized patients with coronavirus disease 2019 in Wuhan, China. JAMA Neurol. 2020;77(6):683-690. doi:10.1001/jamaneurol.2020.1127

15. Romero-Sanchez CM, Diaz-Maroto I, Fernandez-Diaz E, et al. Neurologic manifestations in hospitalized patients with COVID-19: the ALBACOVID registry. Neurology. 2020;95:e1060-e1070. doi:10.1212/WNL.0000000000009937

16. Nalleballea K, Onteddua SR, Sharmaa R, et al. Spectrum of neuropsychiatric manifestations in COVID-19. Brain Behav Immun. 2020;88:71-74. doi:10.1016/j.bbi.2020.06.020

17. Anand P, Zhou L, Bhadelia N, Hamer DH, Greer DM, CervantesArslanian AM. Neurologic findings among inpatients with COVID-19 at a safety-net U.S. hospital. Neurol Clin Pract. 2021;11:e83-e91. doi:10.1212/CPJ.0000000000001031

18. Paniz-Mondolfi A, Bryce C, Grimes Z, et al. Central nervous system involvement by severe acute respiratory syndrome coronavirus-2 (SARS-CoV-2). J Med Virol. 2020;92(7):699-702. doi:10.1002/ jmv.25915

19. Kumar M, Thakur AK. Neurological manifestations and comorbidity associated with COVID-19: an overview. Neurol Sci. 2020;41 (12):3409-3418. doi:10.1007/s10072-020-04823-6

20. Liotta EM, Batra A, Clark JR, et al. Frequent neurologic manifestations and encephalopathy-associated morbidity in Covid-19 patients. Ann Clin Transl Neurol. 2020;7(11):2221-2230. doi:10.1002/ acn3.51210

21. Moein ST, Hashemian SM, Mansourafshar B, Khorram-Tousi A, Tabarsi P, Doty RL. Smell dysfunction: a biomarker for COVID-19. Int Forum Allergy Rhinol. 2020;10(8):944-950. doi:10.1002/ alr. 22587

22. Li Y, Wang M, Zhou Y, et al. Acute cerebrovascular disease following COVID-19: a single center, retrospective, observational study. Stroke Vasc Neurol. 2020;5(3):279-284. doi:10.1136/svn-2020000431

23. Al-Samkari H, Karp Leaf RS, Dzik WH, et al. COVID and coagulation: bleeding and thrombotic manifestations of SARS-CoV2 infection. Blood. 2020;136(4):489-500. doi:10.1182/blood.202 0006520

24. Li MY, Li L, Zhang Y, Wang XS. Expression of the SARS-CoV-2 cell receptor gene ACE2 in a wide variety of human tissues. Infect Dis Poverty. 2020;9(1):45. doi:10.1186/s40249-020-00662-х

25. Connors JM, Levy JH. COVID-19 and its implications for thrombosis and 473 anticoagulation. Blood. 2020;135(23):2033-2040. doi:10.1182/blood.2020006000

26. Reddy ST, Garg T, Shah C, et al. Cerebrovascular disease in patients with COVID-19: a review of the literature and case series. Case Rep Neurol. 2020;12(2):199-209. doi:10.1159/000508958

27. Wang L, He W, Yu X, et al. Coronavirus disease 2019 in elderly patients: characteristics and prognostic factors based on 4-week follow-up. $J$ Inf Secur. 2020;80:639-645. doi:10.1016/j.jinf.20 20.03.019

28. Saavedra JM. Brain angiotensin II: new developments, unanswered questions and therapeutic opportunities. Cell Mol Neurobiol. 2005;25 (3-4):485-512. doi:10.1007/s10571-005-4011-5

29. Niazkar HR, Zibaee B, Nasimi A, Bahri N. The neurological manifestations of COVID-19: a review article. Neurol Sci. 2020;41 (7):1667-1671. doi:10.1007/s10072-020-04486-3

30. Poyiadji N, Shahin G, Noujaim D, Stone M, Patel S, Griffith B. COVID-19-associated acute hemorrhagic necrotizing encephalopathy: imaging features. Radiology. 2020;296:E119-E120. doi:10.1 148/radiol.2020201187

31. Garg RK, Paliwal VK, Gupta A. Encephalopathy in patients with COVID-19: a review. J Med Virol. 2021;93:206-222. doi:10.1002/ jmv. 26207
32. Duong L, Xu P, Liu A. Meningoencephalitis without respiratory failure in a young female patient with COVID-19 infection in Downtown Los Angeles, early April 2020. Brain Behav Immun. 2020;87:33. doi:10.1016/j.bbi.2020.04.024

33. Lv P, Peng F, Zhang Y, et al. COVID-19-associated meningoencephalitis: a care report and literature review. Exp Ther Med. 2021;21:1. doi:10.3892/etm.2021.9793

34. Rahman A, Niloofa R, De Zoysa IM, et al. Neurological manifestations in COVID-19: a narrative review. SAGE Open Med. 2020;8:1-10. doi:10.1177/2050312120957925

35. Huo L, Xu KL, Wang H. Clinical features of SARS-CoV-2-associated encephalitis and meningitis amid COVID-19 pandemic. World J Clin Cases. 2021;9(5):1058-1078. doi:10.12998/wjcc.v9.i5.1058

36. Karimi N, Sharifi Razavi A, Rouhani N. Rouhani NJIRCMJ. Frequent convulsive seizures in an adult patient with COVID-19: a case report. Iran Red Crescent Med J. 2020;22. doi:10.5812/ircmj.102828

37. Lu L, Xiong W, Liu D, et al. New-onset acute symptomatic seizure and risk factors in Corona virus disease 2019: a retrospective multicenter study. Epilepsia. 2020;61(6). doi:10.1111/epi.16524

38. Emami A, Fadakar N, Akbari A, et al. Seizure in patients with COVID-19. Neurol Sci. 2020;41(11):3057-3061. doi:10.1007/ s10072-020-04731-9

39. Zhao H, Shen D, Zhou H, Liu J, Chen S. Guillain-Barré syndrome associated with SARS-CoV-2 infection: causality or coincidence? Lancet Neurol. 2020;19(5):383-384. doi:10.1016/S1474-4422(20) 30109-5

40. Munz M, Wessendorf S, Koretsis G, et al. Acute transverse myelitis after COVID-19 pneumonia. J Neurol. 2020;267(8):2196-2197. doi:10.1007/s00415-020-09934-w

41. Sarma D, Bilello LA. A case report of acute transverse myelitis following novel coronavirus infection. Clin Pract Cases Emerg Med. 2020;4(3):321-323. doi:10.5811/cpcem.2020.5.47937

42. Finsterer J, Scorza FA, Fiorini AC. SARS-CoV-2 associated GuillainBarre syndrome in 62 patients. Eur J Neurol. 2020. doi:10.1111/ ene. 14544

43. Favas TT, Dev P, Chaurasia RN, et al. Neurological manifestations of COVID-19: a systematic review and meta-analysis of proportions. Neurol Sci. 2020;21:1-34. doi:10.1007/s10072-020-04801-y

44. Zhang H, Charmchi Z, Seidman RJ, Anziska Y, Velayudhan V, Perk J. COVID-19-associated myositis with severe proximal and bulbar weakness. Muscle Nerve. 2020;62(3):E57-E60. doi:10.1002/mus.27003

45. Saud A, Naveen R, Aggarwal R, Gupta L. COVID-19 and myositis: what we know so far. Curr Rheum Rep. 2021;23:63. doi:10.1007/ s11926-021-01023-9

46. Brake SJ, Barnsley K, Lu W, McAlinden KD, Eapen MS, Sohal SS. Smoking upregulates angiotensin-converting enzyme-2 receptor: a potential adhesion site for novel coronavirus SARS-CoV-2 (Covid-19). J Clin Med. 2020;9:841. doi:10.3390/jcm9030841

47. Leung JM, Yang CX, Tam A, et al. ACE-2 expression in the small airway epithelia of smokers and COPD patients: implications for COVID-19. Eur Respir J. 2020;55:2000688. doi:10.1183/ 13993003.00688-2020

48. Cai G, Bossé Y, Xiao F, Kheradmand F, Amos CI. Tobacco smoking increases the lung gene expression of ACE2, the receptor of SARSCoV-2. Am J Respir Crit Care Med. 2020;201:1557-1559. doi:10.1164/rccm.202003-0693LE

49. Russo P, Bonassi S, Giacconi R, Malavolta M, Tomino C, Maggi F. COVID-19 and smoking: is nicotine the hidden link? Eur Respir J. 2020;55:2001116. doi:10.1183/13993003.01116-2020

50. Smith JC, Sausville EL, Girish V, et al. Cigarette smoke exposure and inflammatory signaling increase the expression of the SARS-CoV-2 receptor ACE2 in the respiratory tract. Dev Cell. 2020;53:514-529. doi:10.1016/j.devcel.2020.05.012

51. Tang HY, Chiu DTY, Lin JF, et al. Disturbance of plasma lipid metabolic profile in Guillain-Barre syndrome. Sci Rep. 2017;7:8140. doi:10.1038/s41598-017-08338-7 


\section{Publish your work in this journal}

Neuropsychiatric Disease and Treatment is an international, peerreviewed journal of clinical therapeutics and pharmacology focusing on concise rapid reporting of clinical or pre-clinical studies on a range of neuropsychiatric and neurological disorders. This journal is indexed on PubMed Central, the 'PsycINFO' database and CAS, and is the official journal of The International Neuropsychiatric Association (INA). The manuscript management system is completely online and includes a very quick and fair peer-review system, which is all easy to use. Visit http://www.dovepress.com/testimonials.php to read real quotes from published authors.

Submit your manuscript here: https://www.dovepress.com/neuropsychiatric-disease-and-treatment-journal 